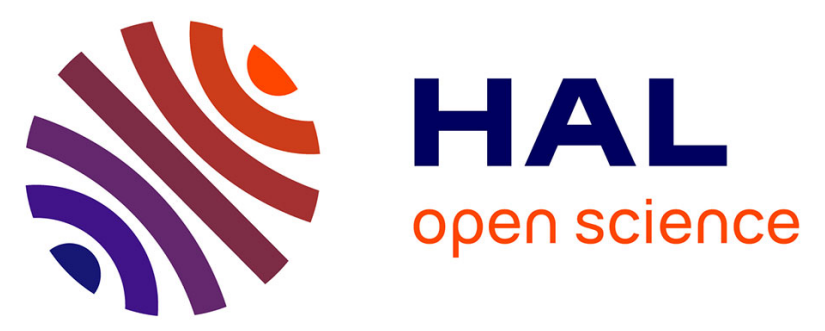

\title{
Automation of ALK gene rearrangement testing with fluorescence in situ hybridization (FISH): A feasibility study
}

Karen Zwaenepoel, Dennis Merkle, Florian Cabillic, Erica Berg, Marc-Antoine Belaud-Rotureau, Vittorio Grazioli, Olga Herelle, Michael Hummel, Michele Le Calve, Dido Lenze, et al.

\section{To cite this version:}

Karen Zwaenepoel, Dennis Merkle, Florian Cabillic, Erica Berg, Marc-Antoine Belaud-Rotureau, et al.. Automation of ALK gene rearrangement testing with fluorescence in situ hybridization (FISH): A feasibility study. Experimental and Molecular Pathology, 2015, 98 (1), pp.113-118. 10.1016/j.yexmp.2015.01.005 . hal-01116397

\section{HAL Id: hal-01116397 \\ https://hal-univ-rennes1.archives-ouvertes.fr/hal-01116397}

Submitted on 22 Oct 2015

HAL is a multi-disciplinary open access archive for the deposit and dissemination of scientific research documents, whether they are published or not. The documents may come from teaching and research institutions in France or abroad, or from public or private research centers.
L'archive ouverte pluridisciplinaire HAL, est destinée au dépôt et à la diffusion de documents scientifiques de niveau recherche, publiés ou non, émanant des établissements d'enseignement et de recherche français ou étrangers, des laboratoires publics ou privés. 
Title

Automation of ALK gene rearrangement testing with Fluorescence in situ Hybridization (FISH): A feasibility study

\section{Authors and affiliations}

Karen Zwaenepoel ${ }^{1, *,+}$, Dennis Merkle ${ }^{2, *}$, Florian Cabillic ${ }^{3}$, Erica Berg ${ }^{4}$, Marc-Antoine Belaud-Rotureau ${ }^{3}$, Vittorio Grazioli ${ }^{5}$, Olga Herelle ${ }^{6}$, Michael Hummel ${ }^{4}$, Michele Le Calve $^{3}$, Dido Lenze ${ }^{4}$, Stefanie Mende ${ }^{4}$, Patrick Pauwels ${ }^{1}$, Benoit Quilichini $^{7}$, Elena Repetti $^{5}$

${ }^{1}$ Antwerp University Hospital, Department of Pathology, Wilrijkstraat 10, 2650, Edegem, Belgium. ${ }^{2}$ Abbott Molecular, Max-Planck-Ring 2, 65205, Wiesbaden, Germany. ${ }^{3}$ University of Rennes 1, Faculty of Medicine, and the Service de Cytogénétique et Biologie Cellulaire, CHU de Rennes, rue Henri Le Guilloux, 35033, Rennes CEDEX 9, France. ${ }^{4}$ Charite University Hospital Berlin, Campus Benjamin Franklin, Hindenburgdamm 30, 12200 Berlin, Germany. ${ }^{5}$ Italian Center of Diagnostics, Laboratory of Molecular Biology and Genetics, Via Saint Bon 20, 20147 Milan, Italy. ${ }^{6}$ Abbott Molecular, Rue de la Couture 12, 94518 Rungis, France. ${ }^{7}$ Biomnis Laboratory, Laboratory of Cytogenetics, 17-19 av Tony Garnier, 69357 Lyon, France.

* KZ and DM contributed equally to this manuscript.

${ }^{+}$Corresponding Author: Karen Zwaenepoel, Antwerp University Hospital, Department of Pathology, Wilrijkstraat 10, 2650 Edegem, Belgium. Phone: +32 3 8214755, Fax: +32 3 8214753 E-mail: Karen.Zwaenepoel@uza.be

Short title: ALK FISH semi-automation 


\section{Abstract}

In the past several years we have observed a significant increase in our understanding of molecular mechanisms that drive lung cancer. Specifically in the non-small cell lung cancer sub-types, ALK gene rearrangements represent a sub-group of tumors that are targetable by the tyrosine kinase inhibitor Crizotinib, resulting in significant reductions in tumor burden. Phase II and III clinical trials were performed using an ALK break-apart FISH probe kit, making FISH the gold standard for identifying ALK rearrangements in patients. FISH is often considered a labor and cost intensive molecular technique, and in this study we aimed to demonstrate feasibility for automation of ALK FISH testing, to improve laboratory workflow and ease of testing. This involved automation of the pre-treatment steps of the ALK assay using various protocols on the VP 2000 instrument, and facilitating automated scanning of the fluorescent FISH specimens for simplified enumeration on various backend scanning and analysis systems. The results indicated that ALK FISH can be automated. Significantly, both the Ikoniscope and BioView system of automated FISH scanning and analysis systems provided a robust analysis algorithm to define ALK rearrangements. In addition, the BioView system facilitated consultation of difficult cases via the internet.

Keywords: Fluorescence in situ hybridization, FISH, VP 2000, automation, BioView, ALK 


\section{Introduction}

In recent years there has been a dramatic increase in the identification of oncogenic driver mutations in non-small cell lung cancer (NSCLC) ${ }^{1}$. In the non-squamous NSCLC subtype two of these driver mutations; epidermal growth factor receptor (EGFR) and the anaplastic lymphoma kinase (ALK), can be targeted by the administration of small molecule tyrosine kinase inhibitors (TKI), gefitinib and erlotinib (for EGFR) ${ }^{2}$ and crizotinib (for ALK) ${ }^{3}$, all of which have demonstrated significant antitumor activity in their respective patient populations $\mathrm{s}^{4-11}$. Specifically for the case ALK gene rearrangements, which were initially discovered in NSCLC in $2007^{12}$, a relatively rapid clinical development occurred, with crizotinib gaining Food and Drug Administration (FDA) approval in the USA in 2011, followed by European Medicines Association (EMA) approval in 2012 (less than five years from discovery to approved therapy). Recently, the College of American Pathologists (CAP), International Association for the Study of Lung Cancer (IASLC) and Association for Molecular Pathology (AMP) published recommendations for detecting ALK gene rearrangements in patients with advanced, nonsquamous NSCLC, to select candidates for crizotinib therapy ${ }^{13}$. Selection of patients for crizotinib TKI therapy should be performed with an ALK fluorescent in situ hybridization (FISH) assay using dual-labelled break-apart probes.

The Vysis ALK Break Apart FISH Probe Kit was utilized to identify ALK rearrangements in NSCLC patients in Phase II and III crizotinib trials, and was co-approved by the FDA as the companion diagnostic for crizotinib therapy selection. While other assay technologies are investigated, including; real-time polymerase chain reaction $(\mathrm{RT}-\mathrm{PCR})^{14,15}$, immunohistochemistry $(\mathrm{IHC})^{16-18}$, and next-generation DNA sequencing $(\mathrm{NGS})^{19,20}$, there is a lack of clinical validation for these technologies in detecting crizotinib responders, and as such, FISH remains the gold standard technology for the detection of ALK rearrangements in NSCLC ${ }^{13,21}$. 
FISH is often considered a complex, and labor intensive molecular technique, and therefore we aimed to study if workflow, and ease of interpretation improvements could be made for the ALK FISH molecular assay by; automating the front-end pretreatment of 20 formalin-fixed paraffin-embedded (FFPE) NSCLC tissue specimens for ALK FISH assaying, as well as, providing automated back-end fluorescence scanning, and interpretation algorithms for enumeration and analysis of the ALK FISH specimens. The pretreatment steps were performed using the VP 2000 instrument, investigating a number of different pretreatment protocols (see materials and methods), were-as the back-end scanning and analysis of ALK FISH cases was investigated by a number of automated fluorescence microscope scanning systems, including; Metasystems, Ikonisys and BioView. The results demonstrated that both front-end and back-end automation of ALK testing are possible, although the specific pretreatment reagents and protocol are critical to obtaining robust results. Different fluorescence scanning systems provide differing levels of interpretation capabilities, from; manual enumeration on scanned images to software assistant interpretation algorithms that are highly concordant with manual reading. Significant workflow possibilities were observed for the BioView instruments, as they allowed web-based consultation and comparison of difficult specimens.

\section{Materials and Methods}

\section{NSCLC Tissue Specimens}

This study was approved by the University Hospital of Antwerp (UZA) Bioethical Committee and includes biopsy and surgical resection samples from 40 patients with advanced NSCLC that were referred for ALK gene rearrangement testing at the institute (UZA) between 2011 and 2013. FFPE tissue blocks were cut on a microtome into serial $5 \mu \mathrm{m}$ sections, floated onto positively charge microscope slides (Thermo Scientific, Waltham, USA), and sent to the four other study sites in Rennes, Berlin, Lyon and Milan. Each site obtained a single 
specimen of each of the 40 patient samples. Prior to pretreatment, the slides were deparaffinated using Xylene on the VP 2000 instrument (Berlin, Lyon) or manually in coplin jars (Antwerp, Rennes and Milan).

Manual FISH staining of NSCLC FFPE specimens- Antwerp

In Antwerp, manual FISH staining of FFPE sections was performed according to the manufacturers recommended protocol using the Vysis Paraffin Pretreatment IV \& PostHybridization Wash Buffer Kit (Abbott Molecular, USA) and the Vysis ALK Break Apart FISH probe kit (Abbott Molecular).

Semi-automated FISH staining of NSCLC FFPE specimens- Rennes, Berlin and Lyon

In Rennes and Berlin the Vysis Paraffin Pretreatment IV \& Post-Hybridization Wash Buffer Kit (Abbott Molecular) was utilized on the VP 2000 instrument (Abbott Molecular), with the protocol as described in the product insert.

In Lyon the VP 2000 Reagents; Pretreatment Reagent, Protease Buffer and Protease II (Abbott Molecular) were utilized on the VP 2000 instrument using following pretreatment protocol: 15 min incubation at $80^{\circ} \mathrm{C}$ with pretreatment solution following by 25 min incubation at $37^{\circ} \mathrm{C}$ using the Protease II solution. Post-hybridization washing was performed using a 0.4XSSC, pH 7/0.3\% NP-40 homebrewed solution (MP Biomedicals, Inc / Abbott Molecular) for 2 min at $73^{\circ} \mathrm{C}$.

Denaturation and hybridization of the Vysis ALK Break Apart FISH probe kit was performed according the instructions insert use using a Thermobrite (Abbott Molecular) in Rennes and Lyon, and on a Dako Hybridizer (Agilent Technologies, Hamburg, Germany) in Berlin. In Berlin, counterstaining was performed using the 4',6-diamidino-2-phenylindole (DAPI) counterstaining from Dako (Agilent Technologies), while Rennes and Lyon utilized the DAPI provided in the Vysis ALK Break Apart FISH probe kit (Abbott Molecular).

Semi-automated FISH staining of NSCLC FFPE specimens- Milan 
In Milan, Italy, a proprietary-homebrew protocol was utilized on the VP 2000. Pretreatment of the specimens occurred at $96^{\circ} \mathrm{C}$ for $15 \mathrm{~min}$ in a TrisEDTA solution (5mM Tris $+1 \mathrm{mM}$ EDTA, pH 7). Pepsin (Porcine gastric mucosa 3555 u, Sigma-Aldrich) treatment $(0.01 \mathrm{~N} \mathrm{HCl}+0.4 \%$ pepsin) was performed for 6 min at $37^{\circ} \mathrm{C}$. Slides were washed in distilled water and let dry. Probes of the Vysis ALK Break Apart FISH probe kit were denatured for 1 min at $85^{\circ} \mathrm{C}$, followed by overnight hybridization at $37^{\circ} \mathrm{C}$ using a Thermobrite (Abbott Molecular). For counterstaining, the Vectashield mounting medium with DAPI (Vector Labs, Peterborough, UK) was used. Post-hybridization washing was performed using a $2 \mathrm{XSSC} / 0.3 \% \mathrm{NP}-40$ homebrewed solution for 2 minutes at $73^{\circ} \mathrm{C}$ (Abbott Molecular).

Interpretation of ALK Fluorescence in situ Hybridization

In all cases, ALK FISH was performed using the Vysis ALK Break Apart FISH probe kit provided by Abbott Molecular. For interpretation of ALK FISH status, the recommended scoring protocol was followed; where specimens were classified as positive for an ALK gene rearrangement (ALK positive) when a minimum of 25 out 50 or a minimum of 15 out of 100 tumor nuclei demonstrated a break apart and/or isolated fluorescent orange FISH signal.

\section{Study Protocol}

As ALK FISH automation was not established prior to the beginning of this study, two sets of specimens were prepared from tissue blocks that had previously been interpreted at the UZA. The first set of specimens contained ten ALK positive and ten ALK negative NSCLC specimens. These twenty specimens were sent to each study site, as a training set, along with the full enumeration and analysis results from Antwerp, in order to allow each lab to develop a back-end automation, or pseudo-automation protocol. Prior to processing the training set, each site ran a set of ProbeChek ALK Negative and ALK Positive Control Slides (Abbott Park, USA) were processed with the VP 2000 at each site. Successful pretreatment of these ProbeChek control slides with VP 2000 allowed each site to proceed with the training set specimens with the same automated protocol. Upon evaluation of the training 
set, and concordant results at each site, a new set of blinded specimens was dispatched to allow for evaluation of the semi-automated protocol. Again at UZA, ten unique ALK positive and ten unique ALK negative NSCLC specimens were prepared. This test set of twenty specimens was then dispatched to each study site, without the results of the analysis. The test set was processed at each site using the respective semi-automation protocol, and results were compared between the sites to gauge the success of each protocol.

\section{Back-end scanning and interpretation of ALK FISH specimens- Antwerp}

At UZA, manual interpretation of the ALK FISH specimens was performed as defined in the Vysis ALK Break Apart FISH probe kit package insert provided by Abbott Molecular on a Olympus BX41 microscope (Olympus, Hamburg, Germany). The specimens were also analyzed on a BioView Allegro-Plus automated scanning and analysis system (Rehovot, Israel) which allowed for automated analysis of ALK FISH. This system is FDA cleared and CE marked for ALK automated imaging and analysis. The system is composed by a fluorescent microscope Olympus BX-61 with a motorized 8-slides stage (Allegro-Plus). Using an $\times 10$ objective and DAPI filter, the operator selected on average 10-20 fields of view (FOV) from each sample. The BioView system performed per FOV automated capture of DAPI, spectrum orange and spectrum green fluorophores in 19 focal layers using single band filters and $x 60$ oil immersion objectives. After completion, the operator reviewed the images and drew circles around 50 tumor nuclei. Each selected nuclei was automatically classified by the BioView software as positive or negative according the signal pattern (defined by probe insert). Next, the operator reviewed the analyzed cells and, if needed, adjusted the classification. Upon evaluation of 50 cells, an automated report can be generated. In case an equivocal result was obtained, 50 additional cells were selected and reviewed by a second analyst.

Back-end scanning and interpretation of ALK FISH specimens- Lyon 
In Lyon, the specimens were analyzed using a BioView automated scanning platform, in analogy to the BioView workflow of Antwerp. The tumors cells were enumerated by the analyst in two ways: manual scoring under microscope eyepiece and automated scoring allowed by Bioview software.

Back-end scanning and interpretation of ALK FISH specimens- Rennes

In Rennes, the specimens were analyzed with a fluorescence microscope Axio Imager Z1 (Zeiss, Göttingen, Germany) and Isis imaging software (Metasystems, Altlussheim, Germany). The entire hybridized surface was screened using a double band-pass filter with an $\mathrm{x} 63$ objective to detect areas with abnormal patterns and to focus the scoring. FISH scoring was performed under both real-time conditions at the microscope and with the use of z-stack images.

Back-end scanning and interpretation of ALK FISH specimens- Berlin

In Berlin, for signal detection and enumeration an Axio Imager Z1 (Zeiss, Jena, Germany) and the Isis software (version 5.3.1, MetaSystems, Altlussheim, Germany) were employed. Images were captured manually and the specimens were then enumerated on a computer screening.

Back-end scanning and interpretation of ALK FISH specimens- Milan

In Milan, the specimens were analyzed using an Ikonisys automated scanning platform, based on the Ikoniscope $囚 /$ lkonisoft Robotic Microscopy instrument (Ikonisys Inc. New Haven, CT USA). The instrument starts with an initial scan using an $\mathrm{x} 4$ objective and DAPI fluorescence channel to identify the tissue section present on the slide. This DAPI tissue image is used to delineate the region of interest, by reference to the H\&E image of the tissue section. Upon scanning this area using a "low magnification" $\times 20$ objective with DAPI, spectrum orange and spectrum green fluorescence channels, the perimeter of each nucleus was delineated and the FISH 3q26 signals in the nucleus were counted. The nuclei were then ordered based on a combination of the number of FISH signals they contained and 
cellular morphology, to select an area of good nuclear distribution representative of the signal distribution observed, for analysis at high magnification. Using a x100 objective 20 fields were then imaged in 11 focal planes with the three fluorescence channels. After nucleus delineation, single orange, single green and paired/fused FISH signals were automatically evaluated according the Vysis ALK Break Apart FISH probe kit package insert. Before a signal was accepted as "true" by the system, three dimensional structure of the potential FISH signals was analyzed for a number of quantitative features. Number and percentage of positive and negative cells were tabulated and presented, along with a gallery of all cells imaged, for expert review. Following confirmation of the 50 cells by the expert, a report was generated. If the result was equivocal, a second set of 50 cells was confirmed for inclusion in the analysis.

Online specimen analysis using the BioView SoloWeb software application

As a number of sites experienced difficulties with the front-end processing of the specimens, a novel FDA cleared web based application online software, SoloWeb, was provided by BioView to allow for remote interpretation of the specimens that were successfully prepared with the manual protocol in UZA, Antwerp, and scanned with the BioView Allegro-Plus system using standard web browsers.

\section{Results}

\section{Training Set}

Twenty NSCLC specimens, ten of which were ALK FISH negative (samples 01-10), and ten which were ALK FISH positive (sample 11-20), were processed manually in Antwerp. Serial sections of the same specimens were dispatched to four different European centers (Rennes, Lyon, Milan and Berlin) for automated front-end specimen processing with VP 
2000, followed by manual analysis. Table 1 shows the results of the twenty training specimens at each site.

Using the manual protocol, UZA demonstrated a $100 \%$ success rate on the samples processed. This was not the case for Rennes, Lyon and Berlin who could only process 12 , 13 and 17 of the 20 training set specimens, respectively. Milan had a $100 \%$ success rate, but did not report the results of the enumeration, as they utilized the results of Antwerp, as a benchmark to develop an automated ALK scoring algorithm on the Ikonisys system.

The results indicated a high degree of variability in technical performance between the various protocols at each site. While the manual ALK FISH protocol demonstrated a robust and reproducible specimen pretreatment, the VP 2000 protocols utilized in Rennes and Lyon demonstrated significant FISH failure rates of $40 \%$ and $35 \%$, respectively. Interestingly, in Berlin, where a nearly identical protocol was utilized as in Rennes, the FISH failure rate on the training set was merely $15 \%$. In Milan, where a proprietary homebrew protocol was employed on the VP 2000, all specimens were processed successfully as judged from examination of FISH signals. Manual enumeration was not performed as the Milano lab sent the specimens to Ikonisys in order to build an automated analysis algorithm for use on the subsequent test set.

\section{Test Set}

As the training set had been deployed to assess the success of the front-end pretreatment protocol, and to allow for training of a back-end system, a new set of twenty blinded, serial NSCLC specimens were sent to each site. These specimens were processed manually in Antwerp, but the results were not shared with the sites. Each site then utilized their respective VP 2000 protocol for the front-end automation, and their respective back-end scanning protocol to elucidate the ALK status of each specimen. 
Again, the manual pretreatment protocol was robust, although two of the ALK negative specimens (24 and 36) failed to processes adequately for interpretation, a consistent observation across all of the sites, except Milan. We observed VP 2000 results consistent to those of the training set in Lyon, Berlin and Rennes, where $30 \%, 20 \%$ and $20 \%$ of the specimens failed pretreatment respectively. In Milan, there was a $100 \%$ success rate on all of the specimens, again confirming the feasibility of a highly robust front-end automation protocol for ALK FISH on the VP 2000 instrument.

With respect to the interpretation of the ALK FISH assay, two of the sites, Rennes and Berlin, utilized scanning systems to capture high resolution images, which were then enumerated manually on computer screens. In Antwerp, Lyon and Milan, fully automated back-end scanning analysis systems were employed.

In Rennes, when the specimens were successfully pretreated, the concordance between positive and negative ALK FISH between the Rennes and Antwerp enumerations was 100\%. In Berlin we observed two disconcordant cases (specimens 27 and 39), which were misclassified as ALK negative. Similar, in Lyon two cases (specimen 25 and 39) were misclassified as ALK negative. Specimen 27 and 39 were the two most challenging ALK positive cases of the test set as they both contained less than $30 \%$ of cells with translocation (as determined by Antwerp, Rennes and Milan).

Using automated analysis algorithms, the BioView system in Antwerp, as well as the Ikonisys system in Milan produced highly concordant results (100\%) for all of the specimens that were successfully pretreated. Interestingly, in Milan, even the two cases that failed pretreatment at the other sites, produced ALK negative results.

Reanalysis of difficult specimens using SoloWeb 
Unfortunately a number of the specimens failed to be processed successfully on the VP 2000 instrument in Lyon, Rennes and Berlin. We wanted to assess if these cases could potentially be remotely analyzed by each site if given access to the raw images utilizing an innovate software developed by BioView, called SoloWeb. The SoloWeb software allowed each of the three sites to log into the BioView captured cases from Antwerp and reinterpret each case over the internet. The results of this reanalysis are also presented in Table 2. With the exception of one case for Lyon (specimen 39) there was $100 \%$ concordance between the interpretations in Antwerp, Rennes, and Berlin. Interestingly, even the two disconcordant cases from Berlin's manual computer image-based read and one of the two disconcordant cases from Lyon's BioView based analysis, were correctly reclassified as ALK positive using the BioView-based analysis algorithms on the images of the tumor regions selected by Antwerp.

\section{Discussion}

In this study, we evaluated the feasibility for ALK FISH automation by comparing results of manual testing with those of automated testing. In a first phase of the study, 20 slides were sent to four European laboratories to assess the success of the front-end pretreatment protocol, and to allow for training of a back-end system. In a second phase, 20 blinded slides were sent to the participating laboratories to access their respective back-end scanning protocols.

Taken together, the results of this study suggest that automation of ALK FISH on the VP 2000 instrument is feasible; however the choice of pretreatment chemistry is critical to obtaining robust results, as the manual protocol reagents for NSCLC could not simply be adapted for the VP 2000 (23.75\% failure), nor were the existing Abbott VP 2000 reagents at the time the study was performed suitable for lung specimens ( $30 \%$ failure). Meanwhile Abbott introduced a new VP 2000 Pretreatment kit optimized for use with lung tissue. Results 
from Milan suggested that some specific pretreatment protocols on the VP 2000 could yield a robust front-end automation protocol. With respect to the interpretation of specimens successfully processed on the VP 2000, the (manual) interpretation of ALK FISH signals was highly consistent and robust, as only one of the 62 results observed (1.6\%) did not correspond between all sites. These data are in agreement with the error rates observed by European Society of Pathology in two EQA round for ALK testing 22.

Results from the blinded test set suggest using computer assisted diagnostic algorithms to aid in the interpretation of ALK FISH, or manual analyzing captured images on a computer screen, can yield highly concordant results $(93.6 \% ; 88$ of 92 informative analysis) to manual interpretation at a microscope in a dark room. We did observe a slight trend for the automated analysis systems to produce slightly higher percentages of ALK positive nuclei as opposed to the manual reading of the specimens; however this did not impact the overall classification of the specimens as ALK positive or negative.

With the SoloWeb software and raw images obtained in Antwerp, remote analysis of the specimens who failed successful processing on the VP 2000 was performed in Rennes, Berlin and Lyon. As the SoloWeb software allows for sharing of images between international sites this software has the potential to serve as a collaborative or consultative tool. Similar to the SoloWeb software of BioView, Ikonisys offers a proprietary InoniWAN server for consultation and/or collaboration.

During the remote analysis, two disconcordant cases from Berlin's manual computer imagebased read and one of the two disconcordant cases from Lyon's BioView analysis, were correctly reclassified as ALK positive, resulting in a concordance of $98.1 \%$ (53 of 54 analysis) compared to manual evaluation. This reduction of incorrect evaluations (from 6.4 to $1.9 \%$ ) illustrates the importance of selecting suited areas for analysis when performing automated analysis. During manual analysis, the analyst selects tumor areas (at lower magnification) and analysis the cells in this area (at high magnification) almost simultaneously. During 
automated processing, these processes are separated in time. As the analyst cannot depend on (his short term memory of) the low magnification image to select tumor cells in the high magnification view, the selection of regions with high tumor content (at low magnification) has become crucial for correct automated ALK FISH analysis. To avoid this issue, both the BioView system as the Ikoniscope platform allow for scanning of the H\&E slide and automatching of the H\&E image to the 10x / 4x DAPI image of the FISH slide respectively. For challenging cases, this workflow is recommended, as this information facilitates the selection of the correct cell population by the analyst.

Taken together this multicenter study demonstrates feasibility that the ALK FISH protocol can be semi-automated from the front-end processing, to the back-end interpretation and image capture of the specimens. Computer assisted software algorithms can aid in the interpretation of ALK FISH specimens. This opens the door to automation of other relevant FISH analysis on FFPE material (e.g. HER2 amplification in breast pathology and ROS1 translocation in lung pathology).

\section{Acknowledgments}

We thank Abbott Molecular for providing the reagents (Vysis Paraffin Pretreatment IV \& PostHybridization Wash Buffer Kit; VP 2000 Reagents and Vysis ALK Break Apart FISH probe kit) to perform the ALK FISH staining at the five sites. We thank BioView for granting us access to the online software, SoloWeb.

\section{Financial Disclosure}

At the time of the study, Dennis Merkle was an employee and shareholder of Abbott Molecular. Olga Herelle is an employee of Abbott Molecular. All other authors have no conflict of interest related to this study. 


\section{References}

1. Chen Z, Fillmore CM, Hammerman PS, Kim CF, Wong KK, 2014. Non-small-cell lung cancers: a heterogeneous set of diseases. Nat Rev Cancer. 14:535-546

2. Bronte G, Rolfo C, Giovannetti E, Cicero G, Pauwels P, Passiglia F, Castiglia M, Rizzo S, Vullo FL, Fiorentino E, Van Meerbeeck J, Russo A, 2014. Are erlotinib and gefitinib interchangeable, opposite or complementary for non-small cell lung cancer treatment? Biological, pharmacological and clinical aspects. Crit Rev Oncol Hematol. 89:300-313

3. Solomon B, Wilner KD, Shaw AT, 2014. Current status of targeted therapy for anaplastic lymphoma kinase-rearranged non-small cell lung cancer.Clin Pharmacol Ther. 95:15-23

4. Camidge DR, Bang YJ, Kwak EL, lafrate AJ, Varella-Garcia M, Fox SB, Riely GJ, Solomon B, Ou SH, Kim DW, Salgia R, Fidias P, Engelman JA, Gandhi L, Jänne PA, Costa DB, Shapiro GI, Lorusso P, Ruffner K, Stephenson P, Tang Y, Wilner K, Clark JW, Shaw AT, 2012. Activity and safety of crizotinib in patients with ALK-positive non-small-cell lung cancer: updated results from a phase 1 study. Lancet Oncol. 13:1011-1019

5. Shaw AT, Kim DW, Nakagawa K, Seto T, Crinó L, Ahn MJ, De Pas T, Besse B, Solomon BJ, Blackhall F, Wu YL, Thomas M, O'Byrne KJ, Moro-Sibilot D, Camidge DR, Mok T, Hirsh V, Riely GJ, lyer S, Tassell V, Polli A, Wilner KD, Jänne PA, 2013. Crizotinib versus chemotherapy in advanced ALK-positive lung cancer.N Engl J Med. 20:2385-2394

6. Zhou C, Wu YL, Chen G, Feng J, Liu XQ, Wang C, Zhang S, Wang J, Zhou S, Ren S, Lu S, Zhang L, Hu C, Hu C, Luo Y, Chen L, Ye M, Huang J, Zhi X, Zhang Y, Xiu Q, Ma J, Zhang L, You C, 2011. Erlotinib versus chemotherapy as first-line treatment for patients with advanced EGFR mutation-positive non-small-cell lung cancer (OPTIMAL, CTONG-0802): a multicentre, open-label, randomised, phase 3 study. Lancet Oncol. 12:735-742

7. Jänne PA, Wang X, Socinski MA, Crawford J, Stinchcombe TE, Gu L, Capelletti M, Edelman MJ, Villalona-Calero MA, Kratzke R, Vokes EE, Miller VA, 2012. Randomized 
phase II trial of erlotinib alone or with carboplatin and paclitaxel in patients who were never or light former smokers with advanced lung adenocarcinoma: CALGB 30406 trial. J Clin Oncol. 30:2063-2069

8. Rosell R, Carcereny E, Gervais R, Vergnenegre A, Massuti B, Felip E, Palmero R, GarciaGomez R, Pallares C, Sanchez JM, Porta R, Cobo M, Garrido P, Longo F, Moran T, Insa A, De Marinis F, Corre R, Bover I, Illiano A, Dansin E, de Castro J, Milella M, Reguart N, Altavilla G, Jimenez U, Provencio M, Moreno MA, Terrasa J, Muñoz-Langa J, Valdivia J, Isla D, Domine M, Molinier O, Mazieres J, Baize N, Garcia-Campelo R, Robinet G, RodriguezAbreu D, Lopez-Vivanco G, Gebbia V, Ferrera-Delgado L, Bombaron P, Bernabe R, Bearz A, Artal A, Cortesi E, Rolfo C, Sanchez-Ronco M, Drozdowskyj A, Queralt C, de Aguirre I, Ramirez JL, Sanchez JJ, Molina MA, Taron M, Paz-Ares L, 2012. Erlotinib versus standard chemotherapy as first-line treatment for European patients with advanced EGFR mutationpositive non-small-cell lung cancer (EURTAC): a multicentre, open-label, randomised phase 3 trial. Lancet Oncol. 13:239-246

9. Maemondo M, Inoue A, Kobayashi K, Sugawara S, Oizumi S, Isobe H, Gemma A, Harada M, Yoshizawa H, Kinoshita I, Fujita Y, Okinaga S, Hirano H, Yoshimori K, Harada T, Ogura T, Ando M, Miyazawa H, Tanaka T, Saijo Y, Hagiwara K, Morita S, Nukiwa T, 2010. Gefitinib or chemotherapy for non-small-cell lung cancer with mutated EGFR. $\mathrm{N}$ Engl $\mathrm{J}$ Med. $362: 2380-2388$

10. Mitsudomi T, Morita S, Yatabe Y, Negoro S, Okamoto I, Tsurutani J, Seto T, Satouchi M, Tada H, Hirashima T, Asami K, Katakami N, Takada M, Yoshioka H, Shibata K, Kudoh S, Shimizu E, Saito H, Toyooka S, Nakagawa K, Fukuoka M, 2010. Gefitinib versus cisplatin plus docetaxel in patients with non-small-cell lung cancer harbouring mutations of the epidermal growth factor receptor (WJTOG3405): an open label, randomised phase 3 trial. Lancet Oncol. 11:121-128 
11. Gridelli C, De Marinis F, Di Maio M, Cortinovis D, Cappuzzo F, Mok T, 2011. Gefitinib as first-line treatment for patients with advanced non-small-cell lung cancer with activating epidermal growth factor receptor mutation: review of the evidence. Lung Cancer 71:249-257

12. Soda M, Choi YL, Enomoto M, Takada S, Yamashita Y, Ishikawa S, Fujiwara S, Watanabe H, Kurashina K, Hatanaka H, Bando M, Ohno S, Ishikawa Y, Aburatani H, Niki T, Sohara $\mathrm{Y}$, Sugiyama $\mathrm{Y}$, Mano $\mathrm{H}, 2007$. Identification of the transforming EML4-ALK fusion gene in non-small-cell lung cancer.Nature. 448:561-566

13. Lindeman NI, Cagle PT, Beasley MB, Chitale DA, Dacic S, Giaccone G, Jenkins RB, Kwiatkowski DJ, Saldivar JS, Squire J, Thunnissen E, Ladanyi M, 2013. Molecular testing guideline for selection of lung cancer patients for EGFR and ALK tyrosine kinase inhibitors: guideline from the College of American Pathologists, International Association for the Study of Lung Cancer, and Association for Molecular Pathology. J Mol Diagn. 15: 415-453

14. Soda M1, Isobe K, Inoue A, Maemondo M, Oizumi S, Fujita Y, Gemma A, Yamashita Y, Ueno T, Takeuchi K, Choi YL, Miyazawa H, Tanaka T, Hagiwara K, Mano H, 2012. A prospective PCR-based screening for the EML4-ALK oncogene in non-small cell lung cancer. Clin Cancer Res. 18:5682-5689

15. Gruber K, Horn H, Kalla J, Fritz P, Rosenwald A, Kohlhäufl M, Friedel G, Schwab M, Ott G, Kalla C, 2014. Detection of rearrangements and transcriptional up-regulation of ALK in FFPE lung cancer specimens using a novel, sensitive, quantitative reverse transcription polymerase chain reaction assay. J Thorac Oncol. 9:307-315

16. Zwaenepoel K, Van Dongen A, Lambin S, Weyn C, Pauwels P, 2014. Detection of ALK expression in non-small-cell lung cancer with ALK gene rearrangements - comparison of multiple immunohistochemical methods. Histopathology. 65:539-548.

17. Cabillic F1, Gros A, Dugay F, Begueret H, Mesturoux L, Chiforeanu DC, Dufrenot L, Jauffret V, Dachary D, Corre R, Lespagnol A, Soler G, Dagher J, Catros V, Le Calve M, 
Merlio JP, Belaud-Rotureau MA, 2014. Parallel FISH and immunohistochemical studies of ALK status in 3244 non-small-cell lung cancers reveal major discordances. J Thorac Oncol. 9:295-306

18 V Laffert M, Warth A, Penzel R, Schirmacher P, Jonigk D, Kreipe H, Schildhaus HU, Merkelbach-Bruse S, Büttner R, Reu S, Kerler R, Jung A, Kirchner T, Wölfel C, Petersen I, Rodriguez R, Jochum W, Bartsch H, Fisseler-Eckhoff A, Berg E, Lenze D, Dietel M, Hummel M, 2013. Anaplastic lymphoma kinase (ALK) gene rearrangement in non-small cell lung cancer (NSCLC): results of a multi-centre ALK-testing. Lung Cancer. 81:200-206

19. Wu K, Huang RS, House L, Cho WC, 2013. Next-generation sequencing for lung cancer. Future Oncol. 9:1323-1336

20. Abel HJ, Al-Kateb H, Cottrell CE, Bredemeyer AJ, Pritchard CC, Grossmann AH, Wallander ML, Pfeifer JD, Lockwood CM, Duncavage EJ, 2014. Detection of gene rearrangements in targeted clinical next-generation sequencing. J Mol Diagn. 16:405-417

21. Thunnissen E, Bubendorf L, Dietel M, Elmberger G, Kerr K, Lopez-Rios F, Moch H, Olszewski W, Pauwels P, Penault-Llorca F, Rossi G, 2012. EML4-ALK testing in non-small cell carcinomas of the lung: a review with recommendations. Virchows Arch. 461:245-257

22. Tembuyser L, Tack V, Zwaenepoel K, Pauwels P, Miller K, Bubendorf L, Kerr K, Schuuring E, Thunnissen E, Dequeker EM, 2014. The Relevance of External Quality Assessment for Molecular Testing for ALK Positive Non-Small Cell Lung Cancer: Results from Two Pilot Rounds Show Room for Optimization. PLoS One. 9:e112159 
Tables

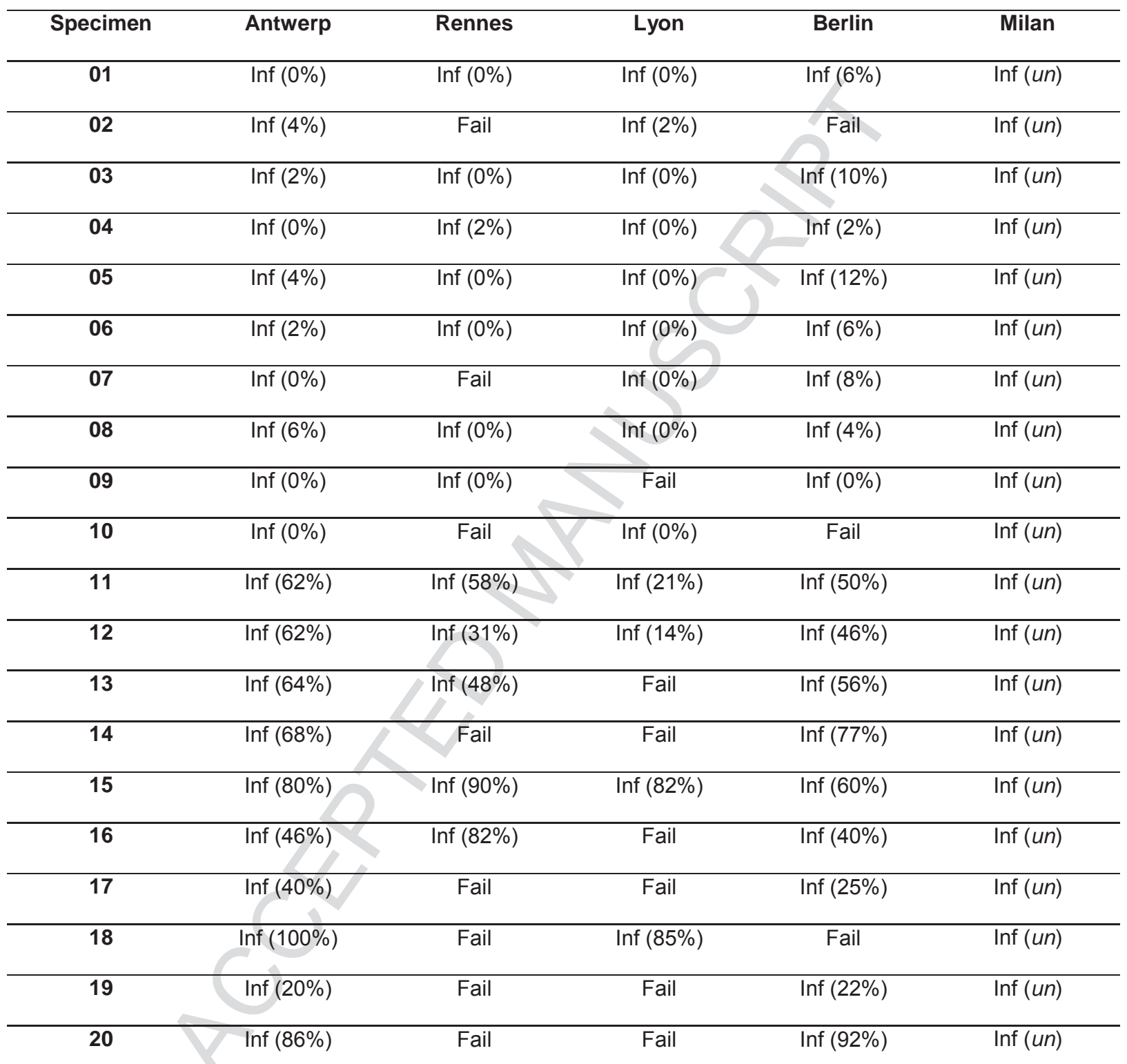

Abbreviations: Inf = informative result, fail = uninformative result, $u n=$ undefined

Table 1: ALK FISH results of training set specimens. Between brackets, the percentage of ALK FISH positive cells observed for each specimen, are represented. 


\begin{tabular}{|c|c|c|c|c|c|c|c|c|c|}
\hline \multirow[b]{2}{*}{ Specimen } & \multirow{2}{*}{$\begin{array}{c}\text { Manual } \\
\text { Antwerp }\end{array}$} & \multicolumn{5}{|c|}{ Automated } & \multicolumn{3}{|c|}{ SoloWeb } \\
\hline & & Rennes & Lyon & Berlin & Milan & Antwerp & Rennes & Lyon & Berlin \\
\hline 21 & 2 & 14 & 0 & 6 & 2 & 6 & 8 & 1 & 8 \\
\hline 22 & 0 & 2 & 0 & 2 & 0 & 2 & 3 & 0 & 2 \\
\hline 23 & 74 & 54 & Fail & Fail & 82 & 90 & 95 & 89 & 94 \\
\hline 24 & Fail & Fail & Fail & Fail & 0 & Fail & Fail & Fail & Fail \\
\hline 25 & 70 & 42 & 0 & 56 & 58 & 53 & 62 & 66 & 76 \\
\hline 26 & 64 & 44 & 70 & 84 & 74 & 80 & 87 & 100 & 96 \\
\hline 27 & 26 & 24 & Fail & 0 & 25 & 44 & 44 & 31 & 74 \\
\hline 28 & 0 & 0 & 0 & 0 & 2 & 14 & 7 & 0 & 4 \\
\hline 29 & 64 & Fail & 50 & 38 & 31 & 63 & 72 & 62 & 64 \\
\hline 30 & 0 & 0 & 0 & 6 & 4 & 4 & 0 & 0 & 4 \\
\hline 31 & 56 & 20 & 65 & 66 & 78 & 80 & 76 & 80 & 70 \\
\hline 32 & 2 & 0 & 0 & 6 & 2 & 4 & 7 & 0 & 4 \\
\hline 33 & 2 & 2 & 0 & 8 & 0 & 8 & 7 & 0 & 4 \\
\hline 34 & 68 & 40 & 70 & 84 & 54 & 90 & 90 & 87 & 82 \\
\hline 35 & 0 & 8 & 0 & 4 & 2 & 12 & 7 & 3 & 14 \\
\hline 36 & Fail & Fail & Fail & Fail & 0 & Fail & Fail & Fail & Fail \\
\hline 37 & 0 & Fail & 0 & Fail & 2 & 4 & 10 & 6 & 8 \\
\hline 38 & 64 & 30 & 80 & 66 & 78 & 96 & 90 & 94 & 78 \\
\hline 39 & 28 & 22 & 0 & 0 & 20 & 68 & 36 & 13 & 44 \\
\hline 40 & 74 & 28 & Fail & 90 & 94 & 96 & 91 & 96 & 97 \\
\hline
\end{tabular}

Table 2: Percentage ALK FISH positivity of test set specimens. Fail indicates that the specimen was not successfully pretreated and did not yield interpretable FISH signals. 